\title{
Electrons and Disorder in Solids
}

\author{
V.F. GANTMAKHER \\ Institute of Solid State Physics, \\ Russian Academy of Sciences, \\ Chernogolovka, 142432 Russia
}

Translated by

Lucia I. Man 


\section{CONTENTS}

1. Metals with strong disorder 1

1.1. Diffraction theory of electron transport in liquid metals 2

$\begin{array}{ll}\text { 1.2. The Mooji rule } & 7\end{array}$

$\begin{array}{lr}\text { 1.3. Saturation of resistivity } & 9\end{array}$

1.4. The Ioffe-Regel limit at high electron density 13

$\begin{array}{ll}\text { References } & 15\end{array}$

2. Quantum corrections to conductivity 16

2.1. Weak localization $\quad 17$

2.2. Effect of magnetic field on weak localization 25

2.3. Antilocalization 28

2.4. Interelectron interference (Aronov-Altshuler effect) 35

References $\quad 41$

3. Effect of the Coulomb interaction on the electron $\begin{array}{ll}\text { energy spectrum } & 43\end{array}$

3.1. The Peierls transition 43

3.2. Structure of the impurity band in the case of low doping 45

3.3. The Coulomb gap $\quad 52$

References $\quad 57$

4. Hopping conductivity 58

4.1. Localized states and transitions between these states 58

4.2. Nearest-neighbor hopping $\quad 61$

4.3. Variable-range hopping $\quad 64$

4.4. Experimental observation of hopping conductivity 67

$\begin{array}{ll}\text { References } & 73\end{array}$

5. Metal-Insulator transitions $\quad 74$

5.1. The Anderson transition $\quad 75$

5.2. The Landauer formula for one-dimensional (1D) systems 78

5.3. Localization and role of correlations in 1D systems 82

$\begin{array}{ll}\text { 5.4. Microwave modelling } & 88\end{array}$

5.5. Model of structural disorder 91

5.6. The Mott transition 94

5.7. The minimum metal conductivity? 99

$\begin{array}{lr}\text { References } & 100\end{array}$

$\begin{array}{ll}\text { 6. Scaling hypothesis } & 102\end{array}$

6.1. Foundations and formulation of the scaling hypothesis 102

$\begin{array}{ll}\text { 6.2. Three-dimensional (3D) systems } & 107\end{array}$ 
6.3. Two-dimensional (2D) systems 114

6.4. Scaling and spin-orbit interaction 120

$\begin{array}{ll}\text { References } & 122\end{array}$

7. Chemical localization 123

7.1. Intermetallic compounds in two-component melts 124

$\begin{array}{ll}\text { 7.2. Quasicrystals } & 130\end{array}$

7.3. Metal-insulator transition in systems with high
electron density

$\begin{array}{ll}\text { References } & 138\end{array}$

8. Granular metals 139

8.1. Morphology and classification 139

8.2. Coulomb blockade and metal-insulator transition 144

8.3. Fractal granular metals 151

$\begin{array}{ll}\text { References } & 155\end{array}$

9. Integer quantum Hall effect 156

9.1. Spectrum and dynamics of two-dimensional electrons
in strong magnetic fields

9.2. Experimental observation of integer quantum Hall effect 164

$\begin{array}{ll}\text { 9.3. Mechanism of plateau formation } & 169\end{array}$

$\begin{array}{ll}\text { 9.4. Edge channels } & 175\end{array}$

9.5. Density of states of $2 \mathrm{D}$ electron gas in a magnetic field 180

9.6. Chains of phase transitions 183

$\begin{array}{ll}\text { 9.7. Two-parametric scaling } & 189\end{array}$

$\begin{array}{ll}\text { References } & 195\end{array}$

$\begin{array}{ll}\text { APPENDICES } & 197\end{array}$

A. Elements of percolation theory $\quad 199$

A.1. Approximation of effective medium 199

$\begin{array}{ll}\text { A.2. Percolation thresholds } & 202\end{array}$

A.3. Region close to the percolation transition 206

$\begin{array}{ll}\text { A.4. Example: Electrical conductivity of a strongly } & \\ \text { inhomogeneous medium } & 208\end{array}$

$\begin{array}{ll}\text { References } & 211\end{array}$

B. Tunneling characteristics $\quad 212$

Materials Index 223

$\begin{array}{ll}\text { Subject Index } & 224\end{array}$ 